\title{
INFLUÊNCIA DO EXERCÍCIO FÍSICO AERÓBICO NA PREVENÇÃO DA DOENÇA CORONARIANA
}

\author{
Reinaldo Bulgarelli Bestetti* \\ José Ernesto dos Santos*
}

\begin{abstract}
BESTETTI, R. B. \& SANTOS, J. E. dos Influência do exercício físico aeróbico na prevenção da doença coronariana. Rev. Saúde públ., S. Paulo, 18:333 - 6, 1984.

RESUMO: A presente revisão enfatiza a função do EFA (Exercício Físico Aeróbico), moderado ou vigoroso, como um importante fator na profilaxia da $\mathrm{AC}$ (arteriosclerose coronariana), sobretudo quando associado a outras medidas profiláticas conhecidas. Esse efeito está provavelmente relacionado com o fato do EFA elevar a concentração de HDL-c. Esta, provavelmente, é a única medida a ser tomada naqueles casos onde não existe nenhum fator de risco associado, mas há forte história familiar de AC. Além do mais, o EFA regular tem importante papel no processo de reabilitação cardíaca nos pacientes portadores de DIM (doença isquêmica do miocárdio).
\end{abstract}

UNITERMOS: Exercício físico aeróbico. Doenças das coronárias.

A hipercolesterolemia constitui fator de risco para o desenvolvimento de arteriosclerose coronariana $(\mathrm{AC})^{1}, 16,17,24$. A hipertrigliceridemia ${ }^{4}$ e a hiperinsulinemia ${ }^{22}$, embora em menor grau, correlacionam-se também, positivamente, com a doença isquêmica do miocárdio (DIM). Estudos clássicos ${ }^{1}$, bem como evidências recentes ${ }^{5}$, mostram que níveis sanguíneos diminuídos de lipoproteinas de densidade alta ou alfa hipoproteínas (HDL-c) ${ }^{* *}$ aumentam o risco de desenvolvimento de $\mathrm{AC}$, enquanto que níveis plas. máticos elevados de HDL-c diminuem a incidência de $\mathrm{AC}$ e aumentam a expectativa de $v^{\text {vida }}{ }^{8}$. Além disso, pacientes que sofreram infarto agudo do miocárdio (IAM) e apresentam baixos níveis de HDL-c (geralmente menor que $35 \mathrm{mg} / 100 \mathrm{ml}$ ) têm pior prognóstico quando comparados com pacientes que tiveram IAM, mas apresentam elevados niveis plasmáticos de $\mathrm{HDL}-\mathrm{c}^{2}$.
Existem vários relatos na literatura médl. $\mathrm{ca}^{6}, 11,12,15,18,20,23,25$ mostrando que o exercício físico aeróbico (EFA) praticario regularmente eleva os níveis plasmáticos de HDL-c. Durante o processo de condicionamento físico observa-se também diminuição significativa nos níveis séricos de triglicerí. $\operatorname{deos}(\mathrm{TG})^{13}$. Isto acontece tanto em indiví. duos submetidos a um processo de condicio. namento físico moderado ${ }^{12}$ como, principalmente, em indivíduos que fazem exercício físico vigoroso ${ }^{3}, 14,18$, como os atletas corredores de grandes distâncias. Nestas pessoas também se observa aumento nos níveis de ácidos graxos livres sanguíneos, refletindo aumento na taxa de lipólise ${ }^{14}$. Esta é provavelmente a explicação para a diminuição dos niveis de TG sanguíneos que se encontra comumente $\mathrm{em}$ individuos bem treinados.

Se existe uma certa concordância na lite-

* Do Departamento de Clínica Médica da Faculdade de Medicina de Ribeirāo Preto da USP - "Campus" de Ribeirão Preto - 14100 - Ribeirão Preto, SP - Brasil.

** Colesterol ligada à alfa lipoproteína. Essa representa a maneira mais freqüentemente utilizada na avaliação dos níveis séricos de alfa hipoproteínas. 
BESTETTI, R. B. \& SANTOS, J. E. dos Influência do exercício físico aeróbico na prevenção da doença coronariana. Rev. Saúde públ., S. Paulo, 18: 333 - 6, 1984.

ratura médica no sentido de que o exercício físico aeróbico reduz os niveis séricos de $\mathrm{TG}$, o mesmo não se pode dizer em relação ao colesterol plasmático (CP). Neste aspecto, a literatura é controvertida. Existem atletas que se submetem a exercício físico vigoroso e não apresentam alterações na colesterole$\mathrm{mia}^{13}$. Entretanto, há relatos de pessoas que fazem exercício físico moderado e apresentam leve, mas significativa diminuição nos niveis de $C P^{12}, 19,20,25$. Portanto, em alguns casos, o EFA pode contribuir para diminuição dos níveis de $\mathrm{CP}$ e, conseqüentemente, para o risco de $\mathrm{AC}$.

O EFA regularmente praticado também provoca uma redução nos níveis séricos de insulina ${ }^{20,}{ }^{23}$, hormônio reconhecidamente lipogênico, cujos níveis são, em geral, elevados em pessoas com $\mathrm{AC}^{22}$.

A maior influência do EFA regular é observada nos níveis sanguíneos de HDL-c Existem relatos convincentes de que ocorre aumento substancial na prevalência de $\mathrm{AC}$ quando os niveis de HDL-c baixam de 53 para $35 \mathrm{mg} / 100 \mathrm{ml}^{21}$. Niveis de HDL-c iguais ou menores que $35 \mathrm{mg} / 100 \mathrm{ml}$ possuem forte correlação com DIM, enquanto que niveis de HDL-c de $45 \mathrm{mg} / 100 \mathrm{ml}$ ou mais reduzem significativamente o risco de $\mathrm{AC}^{10}$. Mais ainda, existem evidências epidemioló. gicas mostrando o desenvolvimento de DIM subseqüentemente em pessoas de meia idade que, aos 20 anos, já apresentavam níveis plasmáticos de HDL-c baixos ${ }^{9}$.

Com base nestas e em citações anteriores verifica-se, claramente, concordância na literatura de que níveis médios ou elevados de HDL-c plasmáticos protegem contra AC. Várias publicações recentes mostram que o EFA regular é capaz de elevar os níveis séricos de HDL-c. Lehtone e Viikari ${ }^{18}$ observaram que os níveis de HDL-c aumentam significativamente em pessoas que correm 25 $\mathrm{km}$ ou mais semanalmente (HDL $=50 \mathrm{mg} / 100$ $\mathrm{ml}$ ), alcançando concentrações excepcionais em individuos que correm $70 \mathrm{~km}$ semanalmente (HDL-c $=70 \mathrm{mg} / 100 \mathrm{ml}$ ). Ao contrário de outros relatos da literatura, estes autores não observaram elevação nos níveis de
HDL-c em individuos que correm menos do que $25 \mathrm{~km}$ por semana, quando comparados a indivíduos não treinados, Huttunen e cols. ${ }^{15}$ submeteram indivíduos saudáveis a um programa de condicionamento físico moderado e também encontraram aumentos significativos nas concentrações séricas de HDL.c. Hartung e cols. ${ }^{11}$ compararam indivíduos que corriam a maratona $(60 \mathrm{~km} / \mathrm{se}$ mana), "joggers" (18 km/semana) e indivíduos saudáveis inativos. Verificaram que os níveis plasmáticos de HDL-c guardavam proporção com o grau de atividade física: os corredores de maratona tinham HDL-c $=65$ $\mathrm{mg} / 100 \mathrm{ml}$, os joggers $=58 \mathrm{mg} / 100 \mathrm{ml}$, en . quanto que os inativos tinham HDL-c $=43$ mg\%. Peltonen e cols. ${ }^{20}$ submeteram um grupo de indivíduos saudáveis a úm programa de condicionamento físico moderado (correr $9 \mathrm{~km} /$ semana, com a $\mathrm{FC} \cong \mathbf{1 5 0} \mathrm{bpm}$ ). Observaram aumento significativo nos niveis séricos de HDL.c. Achados similares foram os de Wood e cols. ${ }^{25}$, quando indivíduos saudáveis que corriam em torno de $24 \mathrm{~km} /$ semana apresentaram níveis de HDL-c de $63 \mathrm{mg} / 100$ $\mathrm{ml}$, enquanto que em indivíduos saudáveis inativos os níveis de HDL-c observados foram de $43 \mathrm{mg} / 100 \mathrm{ml}$.

Esses relatos oferecem subsídios para se concluir que o EFA pode ser uma importante medida profilática contra $\mathrm{AC}$, conforme aumentam os níveis séricos de HDL-c significativamente nas pessoas treinadas, mesmo quando o exercício físico é moderado. A explicação para essa elevação, no entanto, é incerta. E possivel que ela seja decorrente do aumento do transporte de colesterol das células periféricas para o fígado ${ }^{7}$ ou, então, conseqüência da elevação dos níveis de lipase lipoproteina ${ }^{11}$.

Outro aspecto que tem chamado a atenção dos pesquisadores é o efeito do exercício físico regular como medida coadjuvante no processo de reabilitação do paciente com DIM. Erkelens e cols. ${ }^{6}$ submeteram um grupo de pacientes, que sobreviveram a IAM, a um programa de condicionamento físico progressivo, de tal forma que, no último estágio de treinamento, os pacientes corriam 
BESTETTI, R. B. \& SANTOS, J. E. dos Influência do exercício físico aeróbico na prevenção da doença coronariana. Rev. Saúde públ, S. Paulo, 18:333 - 6, 1984.

$2,5 \mathrm{~km}$, três vezes por semana. Verificaram que, em relação a pacientes que sobrevive. ram a IAM, mas não participaram de nenhum tipo de reabilitação cardíaca, ocorreu elevação significativa nos níveis da HDL-c plasmáticos até niveis considerados normais $(\mathrm{HDL}-\mathrm{c}=45 \mathrm{mg} / 100 \mathrm{ml})$.

Resultados similares foram obtidos por Streja e Myamin ${ }^{23}$. Estes dois autores submeteram um grupo de pacientes portadores de DIM (angina ou pós-IAM) a um programa de condicionamento físico moderado (correr $9 \mathrm{~km} / \mathrm{semana}$, com a FC ajustada para $80 \%$ da frequiência cardíaca máxima obtida num teste ergométrico prévio. Nestes pacientes, houve significativa elevação dos niveis séricos de HDL-c $(40 \mathrm{mg} / 100 \mathrm{ml})$. Publicação recente de Heath e cols ${ }^{12}$ também mostra elevação dos níveis de HDL-c $(47 \mathrm{mg} / 100 \mathrm{ml})$ em pacientes portadores de DIM que foram submetidos a um programa de treinamento físico moderado (caminhar, correr ou bicileta estacionária, com $\mathrm{VO}_{2}$ de 50 a $85 \%$ do $\mathrm{VO}_{2}$ máximo previamente calculado). Desta forma, conclui-se que o EFA é importante auxiliar no processo de reabilitação cardíaca de pacientes portadores de AC.

BESTETTI, R. B. \& SANTOS, J. E. dos [The influence of aerobic physical exercise in the prevention of coronary heart disease]. Rev. Saúde públ., S. Paulo, 18: 333 - 6, 1984.

\begin{abstract}
The present review emphazises the role of aerobic physical exercise (APE), of moderate or heavy intensity, as a fundamental tool in the prevention of coronary atherosclerosis (CA). There are evidences that this effect is mediated by the increase of HDL levels. On the other hand increased APE is, in some situations, the only way to prevent CA, specially in patients with no known risk factor but with a considerable family history of CA. In the same way the authors review the importance of APE in the cardiac rehabilitation of patients after myocardiac infarction.
\end{abstract}

UNITERMS: Respiratory exercises. Coronary disease.

\title{
REFERÊNCIAS BIBLIOGRÁFICAS
}

1.BARR, D. P.; RUSS, E. M. \& EDER, H. A. Protein-lipid relationship in human plasma. Amer. J. Med., 11: 480-93, 1951.

2.BERG, K. G.; CANNER, P. L. \& HAINLINE Jr., A. High-density lipoprotein cholesterol and prognosis after myocardial infarction. Circulation, 66: 1176-8, 1982

3. BJORNTROP, P.; FAHLEN, M.; GRIMBY, G.; GUSTAFSON, A.; HOLM, J.; RENSTRAN, P. \& SCHERSTEN, T. Carbohydrate and lipid metabolism in middle aged physically weel-trained men. Metabolism, 21: 1037-44, 1972.

4. CARLSON, L. A. \& BÖTTEIGER, L. E. Ischaemic heart disease in relation to fasting values of plasma triglycerids and cholesterol. Lancet, 1: 865-8, 1972.

5. CASTELLI, W. P.; DOYLE, J. T.; GORDON, T.; HAMES, C. G.; HJORTLAND, M. C.;
HULLEY, S. B.; KAGAN, A. \& ZUKEL, W. J. HDL cholesterol and other lipids in coronary heart disease. Circulation, 55: 767-72, 1977.

6. ERKELENS, D. W.; ALBERS, J. J.;HAZZARD, W. R.; FREDERICK, R. C. \& BIERMAN, E. L. High density lipoprotein cholesterol in survivors of myocardial infarction. $J$. Amer. med. Ass., 242: 2185-9, 1979.

7.GLOMSET, J. A. The plasma licithin cholesterol acyltransferase reaction. J. Lipid Res., 9: 155-67, 1968.

8.GLUECK, C. J.; FALLAT, R. W. \& MILLET, F. Familial hyperalphalipoproteinemia studies in 18 kindred. Metabolism, 24:1243-65, 1975.

9.GOFMAN, J. W.; YOUNG, W. \& TANDY, R. Ischaemic heart disease, atherosclerosis and longevity. Circulation, 34: 679-97, 1966. 
BESTETTI, R. B. \& SANTOS, J. E. dos Influência do exercício físico aeróbico na prevenção da doença coronariana. Rev. Saúde públ., S. Paulo, 18: 333 - 6, 1984.

10.GORDON, T.; CASTELLI, W. P.; HORTLAND, M. C.; KANNEL, W. B. \& DAUBER, T. R. High-density lipoprotein as a protective factor against coronary heart disease. Amer. $J$. Med., 62: 707-14, 1977.

11. HARTUNG, H.; FOREYT, J. P.; MITCHEL, R. E.; VIOSEK, I. \& GOTTO J., A. M. Relation of diet to high-density lipoprotein cholesterol in middle-aged marathon runners, joggers and inactive men. New Engl. J. Med., 302: 357-61, 1980.

12.HEATH, G. W.; EHSANI, A.; HAGBERG, J. M.; HINDERLITER, J. M. \& GOLÖBERG, A. P. Exercise training improves lipoproteins lipid profiles in patients with coronary artery disease. Amer. Heart J., 105: 889-95, 1983.

13. HOLLOSZY, J. O.; SKINNER, J. S.; TORO, G. \& CURETON, T. K. The effects of 2 six month program of endurance exercise on serum lipids of a middle-aged men. Amer. J. Cardiol., 14: 753-60, 1964.

14.HURTER, R.; PEYMAN, M. A.; SWALE, J. \& BARNETT, C. W. H. Some immediate and long-term effects of exercise on the plasma lipids. Lancet, 2: 671.4, 1972.

15.HUTTUNEN, J. K.; LANSIMIES, E.; VQNTILAINEN, F.; EHNHILM, C.; HIETANEN, E.; PENTTILÃ, I.; SHTONEN, O. \& ROURAMAA, R. Effect of moderate physical exercise on serum lipoproteins. Circulation, 60:1220-9, 1979.

16. KANNEL, W. B.; CASTELLI, W. P. \& GORDON, T. Cholesterol in the prediction of atherosclerosis disease. Ann. int. Med., 90: 85-91, 1979.

17. KANNEL, W. B.; CASTELLI, W. P. \& GORDON, T. Serum cholesterol lipoproteins and of coronary heart disease: the Framin. ghan study. Ann. int. Med., 74: 1-12, 1971.
18. LEHTONE, A. \& VIIKARI, J. Serum triglycerides and cholesterol high-density lipoprotein cholesterol in highsty physically active man. Acta med. scand., 204: 111.4, 1978.

19.MANN, G. C.; GARRET, H. L.; FARHI, A.; MURRAY, H. \& BILLINGS, F. T. Exercise to prevent coronary heart disease. Amer. $J$. Med., 46: 12-17, 1969.

20.PELTONEN, P.; MARMIEMI, J.; HIETANEN, E.; VIRARI, I. \& EHNHOLM, C. Changes in serum lipids lipoproteins and heparin releasable lipolytic during moderate physical training in man: a longitudinal study. Metabolism, 30: 518-26, 1981.

21. RHOODS, C. G.; GULBRANDSEN, C. L. \& KAGAN, A. Serum lipoproteins and coronary heart disease in a population study of Havaii Jayanese men. New Engl. J. Med., 294: 293-8, 1976.

22. STOUT, R. W. The relationship of abnormal circulating insulin levels to atherosclerosis. Atherosclerosis, 27: 1-13, 1977.

23. STREJA, D. \& MYAMIN, D. Moderate exercise and high-density lipoprotein-cholesterol. J. Amer. med. Ass., 242: 2190-2, 1979.

24. TRUETT, J.; CORNFIELD, J. \& KANNEL, W. B. A multivariate analysis of the risk of coronary heart disease in Framinghan. $J$. chron. Dis., 20: 511-24, 1967.

25.WOOD, P. D.; HASKELL, W.; KLEIN, H.; LEWIS, S.; STERN, M. P. \& FARGHAR, J. W. The distribution of plasma lipoproteins in middle-age runners. Metabolism, 25: 1249. $-57,1976$.

Recebido para publicagāo em 03/05/1984. Aprovado para publicação em 12/06/1984. 